\title{
Efeitos de Histórias do Ouvinte sobre o Seguimento de Regras Discrepantes das Contingências
}

\author{
Fernanda Monteiro Lima \\ Universidade da Amazônia, Belém, PA, Brasil \\ Luiz Carlos de Albuquerque ${ }^{1}$ \\ Carla Cristina Paiva Paracampo \\ Universidade Federal do Pará, Belém, PA, Brasil
}

\begin{abstract}
Resumo
Objetivando testar proposição sobre as características críticas da história do ouvinte que podem interferir no seguir regra discrepante, oito universitários foram expostos a um procedimento de escolha segundo o modelo. A tarefa era apontar para os três estímulos de comparação em sequência. Na Fase 1, nenhuma sequência era instruída ou reforçada. A Fase 2 era iniciada com a regra correspondente. As contingências da Fase 2 eram alteradas, sem sinalização, na Fase 3, e as contingências da Fase 3 eram mantidas inalteradas na Fase 4, iniciada com a regra discrepante. Dos oito participantes, seis apresentaram um comportamento independente e dois apresentaram um comportamento dependente de suas consequências imediatas na Fase 3. Dos seis participantes que apresentaram um comportamento independente das consequências imediatas na Fase 3, quatro seguiram a regra discrepante na Fase 4. E dos dois participantes que apresentaram um comportamento dependente das consequências imediatas na Fase 3, todos abandonaram o seguimento da regra discrepante na Fase 4. Sugere-se que a dependência e a independência do comportamento às suas consequências imediatas, antes da apresentação da regra discrepante, são variáveis críticas da história do ouvinte que podem ser utilizadas para se prever a manutenção, ou não, do seguimento subsequente de regra discrepante.
\end{abstract}

Palavras-chave: Comportamento governado por regras, comportamento modelado por contingências, justificativas, consequências imediatas, insensibilidade a contingências.

\section{Effects of Listener's Histories on Following Rules that are Discrepant to Contingencies}

\begin{abstract}
Testing proposition by aiming on the listener's history critical characteristics that may meddle on the discrepant-rule following, eight college students were exposed to a matching to sample procedure. The task was to point to the three comparison stimuli in a sequence. In Phase 1 no sequence was instructed or reinforced. Phase 2 begun with the rule corresponding to the contingencies. Contingencies effective in Phase 2 were shifted without warning in Phase 3. Contingencies in Phase 3 were kept unchanged in Phase 4, which started with the presentation of

Endereço para correspondência: Av. Gov. José Malcher, 163/Apto. 06 - B, Nazaré, Belém, PA, Brasil 66035100. E-mail: 1calbu@ufpa.br, cparacampo@gmail.com e nandalima@hotmail.com

Trabalho baseado nos dados da dissertação de mestrado do primeiro autor (programa de Pós-Graduação em Teoria e Pesquisa do Comportamento da Universidade Federal do Pará) e realizado com auxílio do Conselho Nacional de Desenvolvimento Científico e Tecnológico (CNPq), em forma de concessão de bolsa de produtividade em pesquisa ao segundo e ao terceiro autores.
\end{abstract}


a discrepant rule. Six out of eight participants showed an independent behavior and two out of eight showed a dependent behavior from its immediate consequences in Phase 3. From those six out of eight participants showing an independent behavior, four managed to follow the discrepant-rule in Phase 4. And from the latter two out of eight showing a dependent behavior, all of them abandoned the discrepant-rule following in Phase 4. It is suggested that the behavior's dependence and independence to its immediate consequences, before the discrepant-rule presentation, are critical variables of the listener's history that may be used to foresee maintenance, or not, from the subsequent discrepant-rule following.

Keywords: Rule-governed behavior, contingency shaped behavior, justifications, immediate consequences, insensitivity to contingencies.

\section{Efectos de Historias del Oyente acerca del Seguimiento de las Reglas Discrepantes de las Contingencias}

\section{Resumen}

Con objetivo de probar proposición acerca de variables que pueden interferir acerca del seguimiento de regla, ocho estudiantes universitarios fueron expuestos a un procedimiento de elección de acuerdo con el modelo. La tarea era señalar a los tres estímulos de comparación en secuencia. En Fase 1, ninguna secuencia era enseñada o reforzada. Fase 2 comenzaba con la regla correspondiente a las contingencias programadas. Contingencias de Fase 2 eran cambiadas, sin señalización, en Fase 3, y contingencias de Fase 3 eran conservadas en Fase 4, comenzada con la regla discrepante de las contingencias. De los ocho participantes, seis presentaron un comportamiento independiente y dos presentaron un comportamiento dependiente de sus consecuencias inmediatas en Fase 3. De los seis participantes que presentaron un comportamiento independiente de las consecuencias inmediatas en Fase 3, cuatro seguirán la regla discrepante en Fase 4. Y de los dos participantes que presentaron un comportamiento dependiente de las consecuencias inmediatas en Fase 3, todos abandonaran el seguimiento de regla discrepante en Fase 4. Se sugiere que dependencia y independencia del comportamiento a sus consecuencias inmediatas son variables críticas de la historia del oyente que pueden ser usadas para predicción del mantenimiento, o no, del seguimiento subsiguiente de regla.

Palabras clave: Comportamiento gobernado por reglas, comportamiento modelado por contingencias, justificaciones, consecuencias inmediatas, insensibilidad a contingencias.

Regras são estímulos antecedentes verbais que podem descrever o comportamento e suas variáveis de controle; estabelecer a topografia de comportamentos novos; e, alterar as funções de estímulos, independentemente das consequências imediatas produzidas pelo comportamento e de contiguidade espaço-temporal entre estímulo-comportamento e estímulo-estímulo (Albuquerque, Paracampo, Matsuo, \& Mescouto, 2013). Essa definição difere das três principais definições de regras anteriores registradas na literatura [isto é, difere das definições que indicam que regras são estímulos: especificadores de contingências que funcionam como estímulos discriminativos (Skinner, 1969); anteceden- tes verbais (Zettle \& Hayes, 1982); e, alteradores de função de outros estímulos (Schlinger \& Blakely, 1987)], principalmente, porque enfatiza que regras podem exercer suas funções independentemente das consequências imediatas produzidas pelo comportamento e de contiguidade espaço-temporal entre estímulo-comportamento e estímulo-estímulo (Albuquerque \& Paracampo, in press).

Regras exercem suas funções, devido em parte a variáveis ambientais atuais e a variáveis históricas. As principais variáveis ambientais atuais que podem interferir no controle por regras são os tipos de consequências imediatas produzidas pelo seguimento e pelo não segui- 
mento de regras (reforço positivo, reforço negativo, extinção e punição) e os tipos de justificativas para o seguimento e para o não seguimento de regras (Albuquerque \& Paracampo, in press).

Consequências imediatas são eventos produzidos (contatados) imediatamente pelo comportamento após a sua emissão. Justificativas ${ }^{2}$ são estímulos constituintes de uma regra que podem alterar a função de estímulos, determinar a topografia do comportamento e a sua probabilidade de vir a ocorrer e ser mantido. Regras podem ser sem justificativas (Por exemplo, a regra: "Não vá para $Y$, fique aqui em X") e com jus-

Quatro aspectos devem ser esclarecidos: (a) os eventos futuros relatados em justificativas são estímulos antecedentes verbais constituintes da regra e podem exercer controle sobre o comportamento no momento em que a regra é apresentada, quando o ouvinte entra em contato com a regra (Albuquerque, Silva, \& Paracampo, 2014; Matsuo, Albuquerque, \& Paracampo, 2014); (b) já o evento futuro relatado, em si mesmo, não exerce controle sobre o comportamento porque esse evento não é produzido pelo comportamento no momento em que a regra é apresentada. Quando o evento relatado chega a ser produzido pelo comportamento, ele não é produzido como evento futuro, mas sim como uma consequência imediata do comportamento e é dessa forma que ele pode exercer controle. Desse modo, a regra funcionaria como substituto atual do evento futuro por ela relatado (Albuquerque et al., 2014; Matsuo et al., 2014); (c) um comportamento e um evento futuro podem fazer parte de uma contingência de reforço. Contudo, quando essa relação é descrita para um ouvinte e o comportamento especificado por essa regra ocorre, antes de entrar em contato com o evento relatado, tal comportamento deve ser considerado como controlado por regra (Paracampo, Albuquerque, Mescouto, \& Farias, 2013); e, (d) o evento futuro relatado pela justificativa nem sempre é passível de ser produzido (caso de justificativas que relatam que o seguimento de regra produzirá, por exemplo, a bênção de Deus, um paraíso ou um inferno após a morte, etc.) ou é claramente produzido (caso de justificativas que relatam que o seguimento de regra produzirá, por exemplo, aprovação, admiração, felicidade, segurança, saúde, etc.) pelo comportamento especificado pela regra. Nesses casos, a aprovação é indicada por justificativas e não por consequências imediatas (Albuquerque \& Paracampo, in press; Albuquerque et al., 2014; Matsuo et al., 2014). tificativas (por exemplo, "Não vá para Y, fique aqui em $X$. Em $Y$ você não terá apoio, aqui em $X$ você terá tudo o que precisa e ainda terá a sua família para lhe ajudar"). Nesse exemplo, as justificativas diferenciais para não ir para $\mathrm{Y}$ e para ficar em $\mathrm{X}$ alterariam a probabilidade de a ouvinte ficar em X. Portanto, de modo geral, regras podem evocar e determinar a topografia do comportamento e alterar a função de estímulos. Mas, diferente de quando regras são sem justificativas relatadas, quando regras são com justificativas relatadas, tais justificativas podem selecionar e manter o comportamento (Albuquerque \& Paracampo, in press).

Os principais tipos de justificativas são relatos antecedentes verbais a respeito de:

1. As eventuais consequências do seguimento ou do não seguimento de regras; notadas em relatos que podem indicar se as consequências são aversivas ou reforçadoras, de grande ou de pequena magnitude, próximas ou futuras, passíveis de serem contatadas ou não;

2. A eventual aprovação ou desaprovação do seguimento ou do não seguimento de regra; observada em relatos que podem indicar se o falante ou outras pessoas aprovam, ou não, que a regra seja seguida;

3. A confiança do ouvinte no falante; expressa em relatos, tais como, "Eu acho", "Eu tenho experiência", "Eu estou seguro", "Confie em mim", etc., que podem indicar se as consequências relatadas serão realmente produzidas, ou não, pelo seguimento de regra;

4. A forma da regra; vista em relatos que podem indicar se a regra tem a forma de promessa, ordem, ameaça, acordo, discurso, propaganda, documentário e, lei, dentre outras; e,

5. O que observar: relatos que podem indicar exemplos de comportamentos a serem seguidos e exemplos de comportamentos a não serem seguidos (Albuquerque \& Paracampo, 2017a; Albuquerque \& Paracampo, in press; Albuquerque et al., 2013, Albuquerque et al., 2014; Paracampo et al., 2013; Matsuo et al., 2014).

Por exemplo, um falante pode apresentar a regra: "Não faça o curso $Y$, faça o curso X" e 
acrescentar as seguintes justificativas: "Você vai ver que conseguirá um bom emprego e com um bom salário" (justificativa do Tipo 1); "O seu pai vai ficar muito orgulhoso de você e você vai poder ajudar os outros" (justificativa do Tipo 2); "Eu tenho certeza que você conseguirá um bom emprego, pode confiar" (justificativa do Tipo 3); "Eu te peço" (justificativa do Tipo 4); "Fulano fez esse curso, já está empregado e olhe como ele está bem. Já conseguiu até comprar apartamento" (justificativa do Tipo 5). O que há de comum em todos esses exemplos é que as justificativas são estímulos antecedentes verbais que podem alterar as funções de estímulos e interferir na seleção e manutenção do seguimento e do não seguimento de regras (Albuquerque \& Paracampo, 2017a; Albuquerque \& Paracampo, in press).

Quando o comportamento é controlado por regras, a topografia do comportamento e a sua probabilidade de vir a ocorrer e ser mantido, bem como as funções dos estímulos, são determinadas por justificativas. Já quando o comportamento é controlado por contingências de reforço, são as consequências imediatas que exercem essas funções. Dessa forma, os efeitos de regras com justificativas são similares aos efeitos de contingências de reforço, sejam elas verbais ou não verbais. Diferente de tais contingências, regras com justificativas são definidas por serem estímulos antecedentes verbais e por poderem exercer a função de estabelecer e manter comportamento independentemente das consequências imediatas produzidas pelo comportamento e de contiguidade espaço-temporal entre estímulo-comportamento e estímulo-estímulo ${ }^{3}$ (Albuquerque \& Paracampo, in press).

Essa propriedade definidora de regras com justificativas é também a que diferencia tais regras de mando e autoclíticos. Por definição, regras com justificativas exercem tais funções como variáveis ambientais, isto é, como variáveis independentes. Diferentemente, mando e autoclíticos, por definição, são comportamentos, isto é, são variáveis dependentes. Como comportamento, o efeito do mando sobre o ouvinte é o de produzir o reforço especificado, já o efeito do autoclítico sobre o ouvinte é o de produzir o reforço apropriado.
A manutenção do comportamento de seguir e de não seguir regras pode depender de três principais histórias do ouvinte, que diferem quanto às fontes de controle do comportamento especificado pela regra. Na história de controle por consequências imediatas diferenciais, o comportamento especificado pela regra é colocado sob o controle da regra por suas consequências imediatas diferenciais e não por justificativas. Já na história de controle por justificativas diferenciais, o comportamento especificado pela regra é colocado sob o controle da regra por justificativas diferenciais e não por consequências imediatas. E na história de controle pela interação entre justificativas e consequências imediatas diferenciais, para seguir e para não seguir regra, o comportamento especificado pela regra é colocado sob o controle da regra por essas variáveis combinadas (Albuquerque \& Paracampo, 2017a; Albuquerque \& Paracampo, in press).

No presente estudo, procurou-se identificar quais as características críticas que uma história do ouvinte deve apresentar para poder interferir na manutenção do seguimento subsequente de regras discrepantes. Alguns estudos têm procurado identificar tais características críticas (Albuquerque, Mescouto, \& Paracampo, 2011; Albuquerque, Paracampo, \& Allan, no prelo; Albuquerque, Reis, \& Paracampo, 2006; Albuquerque, Reis, \& Paracampo, 2008; Albuquerque \& Silva, 2006; Albuquerque et al., 2014). Dois aspectos devem ser destacados aqui. Primeiro, em todos os procedimentos descritos a seguir, as regras correspondente e discrepante continham a justificativa do Tipo 2 (relatos a respeito da eventual aprovação ou desaprovação do seguimento ou do não seguimento de regra): "Quando eu mostrar estes objetos para você, você deve fazer o seguinte:", que indicava que o experimentador aprovava o seguimento da regra; e, a justificativa do Tipo 1 (relatos a respeito

Portanto, nos dois casos, a ação do ouvinte que se segue a apresentação de um mando ou de um autoclítico, é a de funcionar como auditório, a de reforçar (ou punir) e/ou a de mediar o reforço do comportamento do falante, seja ele mando ou autoclítico (Albuquerque \& Paracampo, in press). 
das eventuais consequências do seguimento ou do não seguimento de regras): "Fazendo isso, você poderá ganhar pontos, que serão mostrados no contador à sua frente", que indicava que o participante ganharia pontos trocáveis por dinheiro se seguisse a regra. A regra era chamada de mínima ("Aponte com o dedo em sequência para cada um dos três objetos de comparação") por que não especificava qual sequência deveria ser emitida e era sem justificativas claras. A regra era chamada de correspondente quando a consequência imediata produzida pelo comportamento por ela especificado correspondia à justificativa do Tipo 1 constituinte da regra, ou seja, nesse caso, quando o seguimento de regra produzia ponto (trocado por dinheiro no final da pesquisa). E a regra era chamada de discrepante quando a consequência imediata produzida pelo comportamento por ela especificado não correspondia à justificativa do Tipo 1 constituinte da regra, isto é, quando o seguimento de regra não produzia ponto (Albuquerque \& Paracampo, 2017b; Albuquerque \& Paracampo, in press). E segundo, um critério para avaliar a dependência ou a independência do comportamento às consequências imediatas por ele produzidas é verificar se o comportamento ocorre sob o controle (isto é, se ocorre dependentemente) de suas consequências imediatas ou se ocorre não sob o controle (isto é, se ocorre independentemente) de tais consequências. Isso pode ser feito pelo menos de duas maneiras: (a) mantendo inalteradas as contingências de reforço programadas no experimento e manipulando as regras (procedimento alternativo, como os usados nos estudos de Albuquerque, de Souza, Matos, \& Paracampo, 2003; Albuquerque et al., 2006, 2008; Martinez \& Tomayo, 2005); e, (b) mantendo inalteradas as regras e manipulando as contingências de reforço programadas no experimento (procedimento tradicional, como os usados nos estudos de Galizio, 1979; Hayes, Brownstein, Zettle, Rosenfarb, \& Korn, 1986; Martinez \& Tomayo, 2005; Paracampo \& Albuquerque, 2004; Perez, Reis, \& de Souza, 2010; Shimoff, Catania, \& Matthews, 1981; Torgrud \& Holborn, 1990). Assim, o termo independência descreve o comportamento que não está sob o controle de suas consequências imediatas e o termo dependência descreve o comportamento que está sob o controle de suas consequências imediatas, em uma determinada situação particular (Albuquerque et al., 2003; Albuquerque \& Paracampo, 2017b; Albuquerque \& Paracampo, in press).

Por exemplo, Albuquerque et al. (2014), usando um procedimento que combina as características dos procedimentos tradicional e alternativo, compararam os efeitos de duas histórias específicas do ouvinte sobre o seguimento subsequente de regra discrepante. Para tanto, expuseram 10 estudantes universitários a um procedimento de escolha de acordo com o modelo, adaptado do desenvolvido por Albuquerque (1991). Os participantes foram distribuídos em dois grupos, com cinco participantes cada, e expostos a quatro fases. A Fase 1 era constituída de 10 tentativas de linha de base onde nenhuma sequência era reforçada ou instruída. Na Fase 2, a sequência correta era reforçada em esquema de reforço contínuo (CRF) e depois da obtenção de 10 pontos, passava a ser reforçada em esquema razão fixa 2 (FR 2) até a obtenção de 10 pontos, quando essa fase era encerrada. Nas Fases 3 e 4, a sequência correta era reforçada em CRF. Essas duas últimas fases eram encerradas de acordo com um dos seguintes critérios, o que ocorresse primeiro: após a concessão de 10 pontos ou após a ocorrência de 30 tentativas. Os grupos diferiam quanto à forma de estabelecimento da sequência correta (CEF) na Fase 2 (por reforço diferencial no Grupo RD e por regra correspondente no Grupo IN). Na Fase 3, as contingências eram alteradas, sem sinalização, de forma que a nova sequência correta era EFC. Finalmente, na Fase 4, as contingências eram mantidas inalteradas, isto é, a emissão da sequência EFC continuava produzindo ponto e a regra discrepante (especificava que se o participante apontasse na sequência FCE ele ganharia pontos) era introduzida.

No Grupo RD, dos quatro participantes que atingiram o critério de desempenho para o encerramento da Fase 2 e, por essa razão, foram expostos às Fases 3 e 4, três (P12, P13 e P14) apresentaram um comportamento dependente das consequências imediatas programadas na Fase 3. Desses três, um (P12) seguiu e dois (P13 
e P14) deixaram de seguir a regra discrepante na Fase 4 e passaram a emitir o comportamento dependente de suas consequências imediatas. E o único participante (P11) que respondeu independentemente das consequências imediatas programadas na Fase 3, seguiu a regra discrepante na Fase 4. No Grupo IN, quatro dos cinco participantes (P22, P23, P24 e P25) continuaram seguindo regra independentemente das consequências imediatas programadas na Fase 3 (dito de outro modo, apresentaram um comportamento insensível ${ }^{4}$ à mudança nas contingências programadas) e seguiram a regra discrepante na Fase 4. Já o único participante (P21) que deixou de seguir regra e apresentou o comportamento dependente das consequências imediatas programadas na Fase 3 (em outras palavras, o que apresentou um comportamento sensível à mudança nas contingências programadas), abandonou o seguimento da regra discrepante na Fase 4.

Os resultados dos Participantes $\mathrm{P} 13$ e $\mathrm{P} 14$ apoiam a proposição de Torgrud e Holborn (1990) de que é improvável que regras exerçam controle sobre o comportamento quando é demonstrado controle discriminativo pelas contingências de reforço antes da introdução da regra. Também apoiam a proposição de que o seguimento de regra discrepante é improvável de ser mantido quando, antes da apresentação dessa regra, é estabelecido por reforço diferencial um comportamento alternativo ao especificado pela regra discrepante e esse comportamento é mantido em esquema de reforço contínuo (Albuquerque et al., 2006). Mas essas proposições não são suficientes para explicar todos os resultados do estudo em exame.

Pela proposição de Albuquerque e Paracampo (2017b), Albuquerque et al. (no prelo) e Albuquerque et al. (2014) as diferenças entre os comportamentos na Fase 3 podem ter ocorrido

4 O termo insensibilidade foi proposto por Shimoff et al. (1981) para descrever o seguimento de regras que não muda quando as contingências de reforço programadas mudam. Contudo, existem controvérsias sobre a adequação do uso desse termo (ver Albuquerque \& Paracampo, 2017 b). devido, em parte, às diferenças entre as histórias do ouvinte na Fase 2. Por exemplo, na história da Fase 2 do Grupo IN, a justificativa do Tipo 2, ao indicar que o experimentador aprovava o seguimento de regra, era uma variável favorável à manutenção do comportamento independentemente de suas consequências imediatas, inclusive na Fase 3. No Grupo RD, como não havia justificativa, as consequências imediatas diferenciais eram as variáveis que indicavam qual a sequência correta (a que produzia reforço) e quais as sequências incorretas (as que produziam extinção). Dessa forma, tais consequências eram as variáveis favoráveis à manutenção de um comportamento dependente de suas consequências imediatas, inclusive na Fase 3. As diferenças entre os desempenhos na Fase 4, por sua vez, podem ter ocorrido devido em parte às diferenças entre as histórias do ouvinte na Fase 3. Uma evidência disso é que o seguimento da regra discrepante apresentou maior probabilidade de ser mantido, quando o comportamento apresentado antes de o contato do ouvinte com a regra discrepante (na Fase 3) foi mantido independentemente de suas consequências imediatas. E o seguimento da regra discrepante apresentou maior probabilidade de deixar de ocorrer, quando esse comportamento foi mantido dependentemente de suas consequências imediatas. Portanto, os resultados de 8 dos 9 (correspondente a $89 \%$ ) participantes expostos às Fases 2 e 3 replicam resultados de estudos anteriores (Albuquerque et al., 2013; Albuquerque \& Silva, 2006; ver também Albuquerque \& Paracampo, 2017b) e estão de acordo com essa proposição (Albuquerque \& Paracampo, 2017b; Albuquerque et al., no prelo; Albuquerque et al., 2014).

A generalidade dessa proposição poderia ser testada em pesquisas que investigassem os efeitos de histórias prolongadas de reforço do comportamento estabelecido por regra correspondente sobre o seguimento subsequente de regra discrepante. Tais investigações são importantes, principalmente, porque podem contribuir para o esclarecimento do papel das variáveis históricas que interferem no seguimento regras discrepantes. Por exemplo, Albuquerque et al. 
(2008, Experimento 2), investigaram os efeitos de uma história experimental prolongada (encerrada após o fornecimento de 320 reforços programados) de controle pela interação entre consequências imediatas e justificativas para seguir regra correspondente sobre o comportamento subsequente de seguir regra discrepante. Para tanto, expuseram quatro estudantes universitários a um procedimento de escolha de acordo com o modelo, adaptado do desenvolvido por Albuquerque (1991). A sequência correta era reforçada em esquema de reforço contínuo (CRF). Os participantes foram expostos às regras mínima, correspondente e discrepante no início das Fases 1, 2 e 3, respectivamente. A Fase 1 (linha de base) era encerrada após a ocorrência de 10 tentativas, a Fase 2 era encerrada após o fornecimento de 320 pontos e a Fase 3 era encerrada após a ocorrência de 240 tentativas ou após o fornecimento de 80 pontos, o que ocorresse primeiro. Os quatro participantes seguiram a regra correspondente na Fase 2. Na Fase 3, todos deixaram de seguir a regra discrepante e passaram a emitir o comportamento alternativo ao especificado pela regra discrepante, previamente estabelecido pela regra correspondente na Fase 2.

Albuquerque et al. (no prelo) fizeram uma replicação sistemática do estudo de Albuquerque et al. (2008, Experimento 2) com quatro novos estudantes universitários. Diferente do estudo de Albuquerque et al. (2008, Experimento 2), no estudo de Albuquerque et al. (no prelo), a sequência correta era reforçada em esquema de razão fixa 4 (FR 4) e foi testada a dependência do comportamento estabelecido pela regra correspondente às suas consequências imediatas no início da Fase 2. Todos os quatro participantes seguiram a regra correspondente durante a Fase 2 e seguiram a regra discrepante durante a Fase 3 , em mais de $98 \%$ das tentativas de cada fase.

Não estão claras quais as variáveis que poderiam ter contribuído para determinar as diferenças entre os resultados dos estudos de Albuquerque et al. (2008, Experimento 2 - CRF longa) e de Albuquerque et al. (no prelo - FR 4 longa). Uma possibilidade seria dizer que, no estudo de Albuquerque et al. (no prelo - FR 4 longa), os participantes seguiram a regra discrepante na Fase 3 porque o comportamento de seguir regras similares foi reforçado no passado (Skinner, 1974). Contudo, essa história da Fase 2 não foi suficiente para manter o seguimento da regra discrepante na Fase 3 no estudo de Albuquerque et al. (2008, Experimento 2 - CRF longa). Outra possibilidade, então, seria dizer que o seguimento da regra discrepante foi abandonado no estudo de Albuquerque et al. (2008, Experimento 2 - CRF longa) e foi mantido no estudo de Albuquerque et al. (no prelo - FR 4 longa), devido às diferenças entre os esquemas de reforço usados nesses dois estudos (Newman, Buffington, \& Hemmes, 1995). Um problema para essa explicação é que há evidências experimentais mostrando que o seguimento de regra discrepante pode ser mantido mesmo quando, antes de ser exposto à regra discrepante, o participante tem uma história curta de reforço contínuo (encerrada após o fornecimento de 80 reforços) do comportamento estabelecido por regra correspondente (Albuquerque et al., 2006, Experimento 1 - CRF curta). Os resultados dos estudos de Albuquerque et al. (2006, Experimento 1 - CRF curta) e Albuquerque et al. (2008, Experimento 2 - CRF longa), em conjunto, indicam que a extensão da história do ouvinte na Fase 2 do estudo de Albuquerque et al. (2008, Experimento 2 CRF longa), pode ter contribuído para impedir a manutenção do seguimento subsequente da regra discrepante na Fase 3 desse estudo. Contudo, os resultados dos estudos de Albuquerque et al. (2008, Experimento 2 - CRF longa) e Albuquerque et al. (no prelo - FR 4 longa), em conjunto, sugerem que a extensão da história, por si só, não é uma variável suficiente para impedir a manutenção do seguimento subsequente da regra discrepante.

Outra possibilidade seria supor que o seguimento da regra discrepante deixou de ocorrer na Fase 3 do estudo de Albuquerque et al. (2008, Experimento 2), possivelmente, porque o comportamento emitido antes da apresentação da regra discrepante era mantido dependentemente de suas consequências imediatas. Uma evidência é 
que, nesse estudo, o comportamento só foi mantido nas Fases 2 e 3 quando produzia as consequências imediatas programadas (ponto). E que o seguimento da regra discrepante foi mantido na Fase 3 do estudo de Albuquerque et al. (no prelo) possivelmente, porque o comportamento emitido antes da apresentação da regra discrepante era mantido independentemente de suas consequências imediatas. Uma evidência é que o seguimento de regra foi mantido nas Fases 2 e 3 desse estudo independentemente de suas consequências imediatas programadas (Albuquerque et al., no prelo).

Considerando essa análise, no presente estudo, procurou-se fazer uma replicação sistemática do Experimento 2 do estudo de Albuquerque et al. (2008), mas, diferente desse estudo e de forma similar ao estudo de Albuquerque et al. (2014, Grupo IN), o presente estudo foi realizado com o objetivo de testar a dependência do comportamento estabelecido pela regra correspondente às suas consequências imediatas, antes da apresentação da regra discrepante. Mais especificamente, no presente estudo, procurou-se, na Fase 2 , construir uma história idêntica à da Fase 2 do estudo de Albuquerque et al. (2008, Experimento 2 - CRF longa). Na Fase 3, diferente do estudo anterior (Albuquerque et al., 2008, Experimento 2), procurou-se alterar, sem sinalização, as contingências de reforço que estavam em vigor na Fase 2. Na Fase 4, procurou-se introduzir a regra discrepante e manter inalteradas as contingências de reforço que estavam em vigor na Fase 3. Desse modo, se a dependência e a independência do comportamento às suas consequências imediatas, antes da apresentação da regra discrepante, são variáveis críticas da história do ouvinte que podem ser utilizadas para se prever a manutenção, ou não, do seguimento subsequente de regra discrepante, deveria ser esperado que o seguimento da regra discrepante fosse mantido, caso fosse antecedido por uma história de independência, e deixasse de ocorrer, caso fosse antecedido por uma história de dependência do comportamento às suas consequências imediatas. Assim, no presente estudo, procurou-se avaliar experimentalmente essas possibilidades.

\section{Método}

\section{Participantes}

Participaram do experimento oito estudantes universitários, sem experiência prévia neste tipo de pesquisa, de diferentes cursos (exceto o de Psicologia) e matriculados em diferentes semestres, dois homens e seis mulheres, com idades variando entre 18 e 23 anos.

\section{Procedimentos Éticos}

Todos os participantes foram voluntários e assinaram o Termo de Consentimento Livre e Esclarecido que, de modo geral, descrevia que a pesquisa objetivava investigar processos de aprendizagem comuns a todas as pessoas e que caso o participante se sentisse incomodado, por qualquer razão, ele poderia interromper a sua participação na pesquisa a qualquer momento e retirar seu consentimento. O projeto foi aprovado pelo comitê de ética (Protocolo: $\mathrm{N}^{\circ}$ 004/2011$\mathrm{CEP} / \mathrm{NMT}$ ).

\section{Equipamento e Material}

Foi utilizada uma mesa de madeira, medindo $150 \times 78 \times 70 \mathrm{~cm}$. Fixada à mesa, de modo a dividi-la ao meio em todo o seu comprimento, havia um anteparo com espelho unidirecional de 150 x $60 \mathrm{~cm}$, fixado em uma moldura de madeira e localizado $13 \mathrm{~cm}$ acima do tampo da mesa. No centro do anteparo, junto ao tampo da mesa, havia uma abertura retangular. Acima e ao centro dessa abertura havia um contador operado pelo experimentador e com os dígitos voltados para o participante. Visível ao participante estava instalada no anteparo uma lâmpada transparente de 5 watts. Uma etiqueta de papel com a frase impressa "Você ganhou um ponto" estava colocada no anteparo acima dessa lâmpada. Uma lâmpada fluorescente de 15 watts estava instalada na borda superior e ao centro do anteparo. A mesa estava situada no centro de uma sala com refrigeração.

Os estímulos modelo e de comparação eram peças de madeira (blocos lógicos da marca FUNBEC), variando em três dimensões: forma (quadrado, círculo, retângulo e triângulo), cor 
(azul, vermelha e amarela) e espessura (grossa e fina). Essas peças de madeira formavam 40 diferentes arranjos de estímulos, cada arranjo era constituído de um estímulo modelo e três estímulos de comparação. Cada estímulo de comparação apresentava apenas uma dimensão - cor (C), espessura (E), ou forma (F) - em comum com o estímulo modelo e diferia nas demais. Os arranjos de estímulos, previamente preparados, ficavam sobre a mesa, ao lado do experimentador, na ordem em que seriam apresentados em cada tentativa. Os estímulos eram apresentados ao participante através da abertura na base do anteparo divisor da mesa, em uma bandeja de madeira em forma de ' $\mathrm{T}$ '. Na parte final do cabo dessa bandeja, quatro ripas de madeira formavam um quadrado no qual era colocado o estímulo modelo. Na base retangular, dividida por ripas de madeira em três quadrados, eram apresentados os três estímulos de comparação. As respostas de apontar para os estímulos de comparação eram registradas pelo experimentador em um protocolo previamente preparado e eram também gravadas por uma filmadora, para análises posteriores. Os reforçadores utilizados eram pontos, registrados no contador. Cada ponto valia $\mathrm{R} \$ 0,05$ (cinco centavos de real). As regras eram gravadas e a gravação era apresentada ao participante por intermédio de fones de ouvido, conectados ao gravador de áudio.

\section{Procedimento}

Durante a sessão experimental, participante e experimentador ficavam sentados à mesa, de frente um para o outro, separados pelo anteparo divisor da mesa. A lâmpada na borda superior do anteparo ficava constantemente acesa, voltada para o participante, de maneira a assegurar que seu lado apresentasse iluminação em maior intensidade, garantindo que apenas as ações emitidas pelo participante, bem como o arranjo dos estímulos apresentados, pudessem ser observadas através do espelho. As quatro fases do experimento (descritas a seguir) foram realizadas em uma única sessão experimental que durava 90 minutos, aproximadamente. $\mathrm{O}$ experimentador, no início das Fases 1, 2 e 4 apresentava ao participante uma determinada regra e, em seguida, apresentava os arranjos de estímulos. Na Fase 3, não apresentava regras, apresentava apenas os arranjos de estímulos. As fases duravam em média $20 \mathrm{~min}$. O intervalo entre fases era de aproximadamente $3 \mathrm{~min}$.

Em cada tentativa, o experimentador apresentava um dos 40 arranjos de estímulos. $\mathrm{Na}$ presença desses estímulos, o participante deveria apontar para cada um dos três estímulos de comparação em uma dada sequência. Caso a sequência de respostas emitida estivesse de acordo com as contingências de reforço programadas (sequência correta), um ponto era acrescentado no contador, a lâmpada transparente era acesa e apagada, e a bandeja com o arranjo de estímulos era retirada. Caso a sequência de respostas fosse incorreta, a lâmpada transparente não era acesa e a bandeja com o arranjo de estímulos era retirada, sem que fosse acrescentado um ponto no contador. Havia um intervalo variável de aproximadamente $5 \mathrm{~s}$ entre uma tentativa e outra. Os pontos eram registrados cumulativamente no contador.

\section{Orientações Preliminares}

No início do experimento, quando participante e experimentador entravam na sala, a bandeja com um arranjo de estímulos estava sobre a mesa, portanto, visível ao participante. $\mathrm{O}$ experimentador pedia ao participante que se sentasse na cadeira e que colocasse os fones de ouvido. No outro lado da mesa, o auxiliar de pesquisa ligava o gravador. Por meio dos fones, o participante passava a ouvir as seguintes orientações:

Este objeto, aqui em cima, é um modelo. Estes três objetos, aqui em baixo, são para você comparar com o modelo. Nós vamos chamar estes três objetos, aqui em baixo, de objetos de comparação. Observe que cada um destes três objetos de comparação tem uma única propriedade comum ao modelo. Veja. Este só tem a espessura comum ao modelo. Este aqui só tem a cor comum ao modelo. E este aqui só tem a forma igual ao modelo. Durante a pesquisa você poderá ganhar pontos que serão trocados por dinheiro. Quando você ganhar pontos, os pontos sempre aparecerão aqui neste contador. Veja como os pontos aparecem no contador [o 
auxiliar, que se encontrava no outro lado da mesa, acionava o contador e acendia a lâmpada cinco vezes]. Quando você não ganhar pontos, nenhum ponto será acrescentado no contador. Entendeu?

O experimentador, ao lado do participante, sempre apontava com o dedo para cada um dos estímulos a que a gravação se referia. Esse procedimento ocorria apenas no início do experimento.

\section{Regras}

A seguir, o experimentador, separado do participante pelo anteparo com espelho unidirecional, dependendo da fase experimental, entregava ao participante, pela abertura na base do anteparo, uma folha de papel contendo uma das seguintes regras impressas:

\section{Regra Mínima:}

Aponte com o dedo, em sequência, para cada um dos três objetos de comparação".

\section{Regra Correspondente:}

Quando eu mostrar estes objetos para você, você deverá fazer o seguinte: primeiro aponte com o dedo para o objeto de comparação que tem a mesma espessura (E) do objeto modelo. Depois aponte para o objeto de comparação que tem a mesma forma $(\mathrm{F})$ do objeto modelo. Em seguida aponte para o objeto de comparação que tem a mesma cor (C) do objeto modelo. Ou seja, você deve apontar primeiro para a mesma espessura, depois para a mesma forma e em seguida para a mesma cor do objeto modelo. Entendeu? Repita para mim o que você deve fazer. Fazendo isso, você poderá ganhar pontos que serão mostrados no contador à sua frente. Cada ponto que você ganhar será trocado por R $\$ 0,05$ (cinco centavos de real), mas apenas no final da pesquisa.

\section{Regra Discrepante:}

Quando eu mostrar estes objetos para você, você deve fazer o seguinte: primeiro aponte com o dedo para o objeto de comparação que tem a mesma forma (F) do objeto modelo. Depois aponte para o objeto de comparação que tem a mesma cor (C) do objeto modelo. Em seguida aponte para o objeto de comparação que tem a mesma espessura (E) do objeto modelo. Ou seja, você deve apontar primeiro para a mesma forma, depois para a mesma cor e em seguida para a mesma espessura. Entendeu? Repita para mim o que você deve fazer. Fazendo isso, você poderá ganhar pontos que serão mostrados no contador à sua frente, cada ponto que você ganhar será trocado por R $\$ 0,05$ (cinco centavos de real), mas apenas no final da pesquisa.

\section{Forma de Apresentação das Regras}

Imediatamente após entregar ao participante a folha de papel contendo a regra impressa, o experimentador ligava o aparelho de som reprodutor de áudio e o participante passava a ouvir a gravação com a voz do experimentador lendo a regra referente à fase que seria iniciada. $\mathrm{Na}$ gravação, o experimentador solicitava ao participante, ora que acompanhasse a leitura, ora que lesse sozinho, silenciosamente. Após a última leitura, a gravação solicitava ao participante que devolvesse a folha com a regra. Logo após o participante devolver a folha com a regra, o auxiliar de pesquisa removia a bandeja, voltava a apresentar a bandeja com um novo arranjo de estímulos e o experimentador dizia: "Comece a apontar".

\section{Delineamento Experimental}

O experimento era constituído de quatro fases. A Fase 1 era iniciada com a apresentação da regra mínima; a Fase 2, com a regra correspondente; a Fase 3, com a mudança não sinalizada nas contingências programadas; e, a Fase 4, com a regra discrepante.

A Fase 1 era constituída de 10 tentativas de linha de base em relação à qual eram avaliados os efeitos da introdução da regra correspondente na Fase 2. Durante a Fase 1, nenhuma sequência de respostas era reforçada ou descrita por regra. 
Durante as Fases 2, 3 e 4, as sequências corretas eram reforçadas, com pontos trocáveis por dinheiro, em esquema de reforço contínuo.

$\mathrm{Na}$ Fase 2, a única sequência que produzia ponto (correta) era EFC, especificada pela regra correspondente. A Fase 2 era encerrada após a conceção de 320 pontos. Na Fase 3, a sequência EFC (correta na Fase 2) deixava de produzir pontos e a sequência CEF passava a ser a única (correta) que produzia pontos. A Fase 3 era encerrada após a ocorrência de 80 tentativas. $\mathrm{Na}$ Fase 4, a sequência CEF (correta na Fase 3) continuava sendo a única (correta) que produzia pontos. Portanto, a sequência incorreta FCE, especificada pela regra discrepante, não produzia pontos. A Fase 4 também era encerrada após a ocorrência de 80 tentativas.

\section{Comparação dos Registros e Término da Participação do Estudante no Experimento}

Ao final da Fase 4, um observador independente comparava o registro feito pelo experimentador no protocolo com o registro feito pela filmadora. Caso houvesse $100 \%$ de concordância entre os registros, os dados eram considerados para análise. Caso contrário, eram descartados. Nenhum dado foi descartado na presente pesquisa. A participação do estudante no experimento era concluída após ser atingido o critério de encerramento da Fase 4.

\section{Procedimentos de Análise de Dados}

Primeiro, foram analisados os efeitos da história da Fase 3 sobre a manutenção do seguimento de regra discrepante na Fase 4 (transição da Fase 3 para a Fase 4), uma vez que esse é o objetivo do presente estudo. Mas também foram feitas suposições a respeito de efeitos da história da Fase 2 sobre o comportamento na Fase 3 (transição da Fase 2 para a Fase 3).

\section{Resultados}

Serão apresentados os dados para cada fase e serão feitas comparações entre fases. $\mathrm{Na}$ Tabela 1 são apresentadas as porcentagens de se- quências de respostas emitidas durante as quatro fases pelos oito participantes. Pode-se observar que, na Fase 1, os participantes variaram seus desempenhos, apresentando diferentes sequências.

Na Fase 2, quando a regra correspondente foi apresentada, todos os oito participantes seguiram essa regra em $100 \%$ das tentativas. Na Fase 3 , quando as contingências de reforço programadas foram alteradas, seis dos oito participantes (P11, P12, P13, P15, P17 e P18) continuaram emitindo a sequência EFC, previamente especificada pela regra correspondente, isto é, continuaram seguindo regra, apesar de esse comportamento não mais produzir o reforço programado. Diferentemente, P14 e P16 deixaram de seguir a regra e passaram a responder corretamente, emitindo a sequência CEF.

$\mathrm{Na}$ Fase 4, quando a regra discrepante foi introduzida e as contingências de reforço programadas foram mantidas inalteradas, dos seis participantes $(\mathrm{P} 11, \mathrm{P} 12, \mathrm{P} 13, \mathrm{P} 15, \mathrm{P} 17$ e P18) que apresentaram um comportamento independente das consequências imediatas programadas na Fase 3, quatro (P11, P12, P13 e P15) seguiram a regra discrepante e, desse modo, também apresentaram um comportamento independente das consequências imediatas programadas na Fase 4. E dos dois participantes (P14 e P16) que apresentaram um comportamento dependente das consequências imediatas programadas na Fase 3, todos abandonaram o seguimento da regra discrepante e, desse modo, também apresentaram um comportamento dependente das consequências imediatas programadas na Fase 4

O Participante P17, que continuou seguindo regra na Fase 3, deixou de seguir a regra discrepante na Tentativa 59 da Fase 4, emitiu a sequência incorreta CFE e passou a responder corretamente no final da Fase 4. O Participante P18 também continuou seguindo regra na Fase 3 e deixou de seguir a regra discrepante na Fase 4. Depois, passou a apresentar a sequência incorreta CFE. Dessa forma, P18 também apresentou um comportamento independente das contingências de reforço programadas nas Fases 3 e 4. 
Tabela 1

Porcentagens de Sequências Emitidas pelos Participantes (P) durante as Fases 1, 2,3 e 4.

\begin{tabular}{|c|c|c|c|c|c|c|}
\hline Participantes & & Sequê & ias de $R$ & postas & & \\
\hline Fase 1 & EFC & ECF & FCE & FEC & CEF & CFE \\
\hline P11 & 0 & 80 & 10 & 0 & 0 & 10 \\
\hline P12 & 30 & 10 & 20 & 30 & 10 & 0 \\
\hline P13 & 0 & 20 & 10 & 30 & 30 & 10 \\
\hline P14 & 10 & 30 & 10 & 30 & 20 & 0 \\
\hline P15 & 20 & 10 & 0 & 40 & 20 & 10 \\
\hline P16 & 0 & 30 & 20 & 20 & 20 & 10 \\
\hline P17 & 0 & 0 & 40 & 40 & 0 & 20 \\
\hline P18 & 20 & 0 & 20 & 10 & 20 & 30 \\
\hline Fase 2 & EFC & ECF & FCE & FEC & CEF & CFE \\
\hline P11 & $100^{*}$ & 0 & 0 & 0 & 0 & 0 \\
\hline P12 & $100^{*}$ & 0 & 0 & 0 & 0 & 0 \\
\hline P13 & $100 *$ & 0 & 0 & 0 & 0 & 0 \\
\hline P14 & $100 *$ & 0 & 0 & 0 & 0 & 0 \\
\hline P15 & $100^{*}$ & 0 & 0 & 0 & 0 & 0 \\
\hline P16 & $100^{*}$ & 0 & 0 & 0 & 0 & 0 \\
\hline P17 & $100^{*}$ & 0 & 0 & 0 & 0 & 0 \\
\hline P18 & $100^{*}$ & 0 & 0 & 0 & 0 & 0 \\
\hline Fase 3 & EFC & ECF & FCE & FEC & CEF & CFE \\
\hline P11 & 100 & 0 & 0 & 0 & 0 & 0 \\
\hline P12 & 98 & 1 & 0 & 1 & 0 & 0 \\
\hline P13 & 100 & 0 & 0 & 0 & 0 & 0 \\
\hline P14 & 10 & 1 & 1 & 1 & $87^{*}$ & 0 \\
\hline P15 & 99 & 1 & 0 & 0 & 0 & 0 \\
\hline P16 & 9 & 5 & 2 & 0 & $65^{*}$ & 19 \\
\hline P17 & 100 & 0 & 0 & 0 & 0 & 0 \\
\hline P18 & 91 & 3 & 1 & 0 & 0 & 5 \\
\hline Fase 4 & EFC & ECF & FCE & FEC & CEF & CFE \\
\hline P11 & 0 & 0 & $100 * *$ & 0 & 0 & 0 \\
\hline P12 & 0 & 0 & $99 * *$ & 0 & 0 & 1 \\
\hline P13 & 0 & 0 & $100 * *$ & 0 & 0 & 0 \\
\hline P14 & 0 & 0 & $3^{* *}$ & 1 & $96 *$ & 0 \\
\hline P15 & 1 & 0 & $99 * *$ & 0 & 0 & 0 \\
\hline P16 & 0 & 0 & $3^{* *}$ & 0 & $97^{*}$ & 0 \\
\hline P17 & 4 & 1 & $24 * *$ & 1 & $29 *$ & 41 \\
\hline P18 & 1 & 0 & $31^{* *}$ & & $3 *$ & 65 \\
\hline
\end{tabular}

Nota. $\mathrm{C}=$ resposta à dimensão cor; $\mathrm{E}=$ resposta à dimensão espessura; $\mathrm{F}=$ resposta à dimensão forma. *Indica sequência reforçada. **Indica sequência especificada pela regra discrepante. As Fases 1, 2, 3 e 4 eram iniciadas por: regra mínima, regra correspondente; mudança não sinalizada nas contingências programadas; regra discrepante, respectivamente. 


\section{Discussão}

No presente estudo, procurou-se testar a proposição que sugere que a dependência e a independência do comportamento às suas consequências imediatas, antes da apresentação da regra discrepante, são variáveis críticas, constituintes da história do ouvinte, que podem ser utilizadas para se prever a manutenção, ou não, do seguimento subsequente de regra discrepante (Albuquerque \& Paracampo, 2017b; Albuquerque et al., no prelo). Os resultados de 6 de 8 participantes (correspondente a $75 \%$ ) do presente estudo replicam resultados de estudos anteriores (Albuquerque \& Paracampo, 2017b; Albuquerque et al., 2013; Albuquerque et al., 2014; Pinto, Paracampo, \& Albuquerque, 2006) e apoiam essa proposição. Tal como essa proposição sugere, o seguimento da regra discrepante foi provável de ser mantido quando, antes da apresentação dessa regra, o participante apresentou um comportamento mantido independentemente de suas consequências imediatas (caso de P11, P12, P13 e P15); e, o seguimento da regra discrepante foi provável de deixar de ocorrer quando, antes da apresentação dessa regra, o participante apresentou um comportamento mantido dependentemente de suas consequências imediatas (caso de $\mathrm{P} 14$ e P16). Portanto, as diferenças entre seguir e não seguir a regra discrepante dependem em parte das diferenças entre as histórias dos ouvintes (Albuquerque \& Paracampo, 2017b; Albuquerque \& Paracampo, in press; Albuquerque et al., no prelo; Albuquerque et al., 2014).

Um problema, no entanto, consiste em explicar por que os comportamentos dos Participantes P11, P12, P13 e P15 foram mantidos independentemente de suas consequências imediatas e por que os comportamentos dos Participantes P14 e P16 foram mantidos dependentemente de tais consequências na Fase 3? Não está claro por que tais diferenças ocorreram, mas algumas sugestões podem ser oferecidas visando à realização de pesquisas futuras. As diferenças individuais encontradas no presente estudo podem ter ocorrido devido em parte a possíveis diferenças entre as histórias pré-experimentais de seguimento de regras dos participantes, visto que tais diferenças também foram encontradas nos estudos anteriores (Albuquerque et al., 2013, Albuquerque et al., 2014; Pinto et al., 2006). Essa proposição também se baseia em resultados experimentais de alguns estudos que têm investigado os efeitos de histórias pré-experimentais (inferidas das respostas do ouvinte ao questionário a respeito de inflexibilidade desenvolvido por Rehfisch, 1958) sobre o seguimento de regra (Albuquerque \& Paracampo, 2017b; Paracampo, Souza, \& Albuquerque, 2014; Pinto et al., 2006; Wulfert, Greenway, Farkas, Hayes, \& Douguer, 1994). Por exemplo, Pinto et al. (2006) expuseram oito estudantes universitários (quatro flexíveis e quatro inflexíveis) a um procedimento que diferiu do utilizado no Grupo IN do estudo de Albuquerque et al. (2014), principalmente, porque os oito participantes foram pré-selecionados com base em suas respostas ao questionário sobre inflexibilidade. Os cinco participantes (quatro inflexíveis e um flexível) que continuaram seguindo regra na Fase 3 (isto é, que apresentaram um comportamento mantido independentemente de suas consequências imediatas), seguiram a regra discrepante na Fase 4; e os três participantes (todos flexíveis) que deixaram de seguir regra na Fase 3 e passaram a apresentar um comportamento mantido dependentemente de suas consequências imediatas, deixaram de seguir a regra discrepante na Fase 4. Tais resultados, em conjunto com os resultados do presente estudo, indicam que histórias pré-experimentais (inferidas das respostas do ouvinte ao questionário sobre inflexibilidade) podem interferir no comportamento de seguir e de não seguir regra.

Mas os resultados do estudo de Pinto et al. (2006) não indicam qual a história pré-experimental interfere no comportamento, ou seja, não indicam se essa história do ouvinte é de controle (a) por consequências imediatas diferenciais, (b) por justificativas diferenciais ou (c) pela interação entre justificativas e consequências imediatas diferenciais, para seguir e para não seguir regra. Além disso, há evidências experimentais que indicam que os eventuais efeitos de histórias pré-experimentais na determinação de diferenças individuais, dependem em parte de variáveis ambientais atuais à quais os ouvintes são expos- 
tos (Paracampo et al., 2014). Por essa proposição, são as variáveis ambientais atuais que favorecem, ou não, os eventuais efeitos de histórias pré-experimentais na determinação de diferenças individuais (Albuquerque \& Paracampo, 2017b; Albuquerque \& Paracampo, in press; Paracampo et al., 2014). No presente estudo há evidências que apoiam essa proposição.

Por exemplo, os resultados do presente estudo, mostrando que ocorreram diferenças entre os desempenhos dos participantes nas Fases 1, 3 e 4 e que tais diferenças não ocorreram na Fase 2, indicam que, diferente da Fase 2, nas Fases 1,3 e 4 haviam variáveis que poderiam favorecer os efeitos de histórias pré-experimentais na determinação de diferenças individuais. $\mathrm{Na}$ Fase 1, todos seguiram a regra mínima, isto é, tal como especificado por essa regra, todos emitiram o comportamento de apontar com o dedo (e não outro) para cada um dos três estímulos de comparação (e não para outros estímulos). Apontaram em diferentes sequências porque a regra mínima não especificava uma sequência. Uma evidência disso é que essa variabilidade foi eliminada na Fase 2, quando foi introduzida a regra correspondente que especificava a sequência a ser emitida e todos passaram a emitir a sequência especificada. Mas o fato de especificar o comportamento a ser emitido, por si só, não é suficiente para explicar por que não foram observadas diferenças entre os desempenhos dos participantes na Fase 2, uma vez que na Fase 4, a regra discrepante introduzida também especificava tal comportamento e foram observadas tais diferenças. Uma variável que interferiu na ocorrência, ou não, de diferenças entre os desempenhos dos participantes foi a justificativa do Tipo 1 (relatos a respeito das eventuais consequências do seguimento ou do não seguimento de regras): "Fazendo isso, você poderá ganhar pontos que serão mostrados no contador à sua frente". Ou seja, os resultados do presente estudo mostraram que as diferenças entre os desempenhos dos participantes foram mais prováveis de ocorrer quando essa justificativa não correspondia (Fases $3 \mathrm{e}$ 4) do que quando correspondia (Fase 2) às consequências imediatas produzidas pela emissão do comportamento especificado pela regra.
Por essa análise, além das histórias pré-experimentais, variáveis ambientais atuais também podem ter determinado os desempenhos dos participantes. No caso de P14 e P16, o contato do seguimento de regra com a discrepância entre a justificativa do Tipo 1 (a promessa de obtenção de pontos) e as consequências imediatas (a não obtenção de pontos) produzidas por esse comportamento nas Fases 3 e 4, possivelmente, contribuiu para que esses dois participantes (P14 e P16) tivessem deixado de seguir regra nessas fases (Galizio, 1979). A discriminação dessa discrepância pode ter sido facilitada pela diferença entre a história prolongada de reforço contínuo do seguimento de regra na Fase 2 e a extinção produzida por esse comportamento na Fase 3 (Albuquerque et al., 2008; Cerutti, 1989; Newman et al., 1995). O fato de o não seguimento de regra ter sido reforçado em esquema de reforço contínuo também pode ter favorecido o abandono do seguimento de regra (Paracampo \& Albuquerque, 2004).

Um problema, no entanto, consiste em explicar por que, mesmo sendo expostos a essas variáveis que favoreciam o abandono do seguimento de regra, os Participantes P11, P12, P13 e P15 continuaram seguindo regra na Fase 3? Para tentar esclarecer essa questão é necessário também considerar os efeitos, sobre o seguimento de regra, da justificativa do Tipo 2 (relatos a respeito da eventual aprovação ou desaprovação do seguimento ou do não seguimento de regra): "Quando eu mostrar estes objetos para você, você deve fazer o seguinte:”. Isso é necessário porque há evidências experimentais que mostram que exemplos de justificativas do Tipo 2 que indicam que o falante aprova ou faz questão que a regra seja seguida, tal como a que foi apresentada aos participantes no presente estudo, têm maior probabilidade de manterem o comportamento especificado por regras discrepantes do que justificativas do Tipo 2 que não indicam claramente que o falante aprova ou faz questão que a regra seja seguida.

Por exemplo, há evidências experimentais (Albuquerque et al., 2011) mostrando que o comportamento subsequente especificado pela regra discrepante apresentou maior probabilidade de 
ser instalado e mantido, quando a regra discrepante continha a justificativa do Tipo 2: "Quando eu mostrar estes objetos para você, eu quero que você faça o seguinte:..." (para os autores, exemplos de justificativa do Tipo 2 como esse, poderiam indicar que o seguimento de regra está sendo monitorado, que o falante se importa com o seguimento de regra e que o não seguimento de regra implica em claramente desobedecer o falante) do que quando tal regra continha a justificativa do Tipo 2: "Quando eu mostrar estes objetos para você, faça o que achar melhor para você. Se você quiser, você pode fazer o seguinte:...". (para os autores, exemplos de justificativa do Tipo 2 como esse, poderiam indicar que o seguimento de regra não está sendo monitorado, que o ouvinte não é obrigado a seguir a regra e, portanto, que o não seguimento de regras não seria punido pelo falante). Tais resultados sugerem que os efeitos de justificativas deveriam passar a ser considerados na explicação da manutenção do comportamento especificado por regras (ver também Albuquerque \& Paracampo, in press).

De acordo com Albuquerque et al. (2014), os efeitos de justificativas, no entanto, ao invés de serem considerados efeitos de estímulos antecedentes verbais (isto é, de estímulos constituintes de regras), têm sido considerados como se fossem efeitos de consequências imediatas (isto é, de contingências de reforço), ou mais especificamente, como se fossem efeitos de: contingências verbais (Skinner, 1969); consequências mediadas socialmente (Hayes et al., 1986; Zettle \& Hayes, 1982); consequências instrucionais (Cerutti, 1989); consequências culturais (Matos, 2001); contingências que agem direta e indiretamente (Malott, 1989); contingência próxima e contingência última (Baum, 1994/1999); e, contingências verbais e sociais de ordem superior (Catania, 1998). O problema do uso de tais termos é que eles não contribuem para o esclarecimento da distinção entre o que é controle por estímulos constituintes de regras e o que é controle por estímulos constituintes de contingências de reforço e, dessa maneira, não contribuem para distinguir o que é controle por regra e o que é controle por contingências. Ainda de acordo com Albuquerque et al. (2014), o falante pode indicar para o ouvinte que aprova ou desaprova o responder de acordo com uma regra por justificativas ou por consequências imediatas. A diferença é que os estímulos (como críticas, elogios, indicações de admiração, de rejeição, de comportamento que é correto ou incorreto, etc.) são apresentados antes da ocorrência do comportamento, no primeiro caso, e imediatamente após a ocorrência do comportamento, no segundo caso (ver também Albuquerque \& Paracampo, in press).

Por essa proposição, os resultados do presente estudo, em conjunto com os resultados de estudos similares (Albuquerque et al., 2011; Albuquerque et al., no prelo; Albuquerque et al., 2014), sugerem que, na Fase 2 do presente estudo, os Participantes P11, P12, P13 e P15 seguiram a regra, possivelmente, mais sob o controle da justificativa do Tipo 2 (relatos a respeito de eventual aprovação, ou não, do seguimento de regra) do que da correspondência entre a justificativa do Tipo 1 (relatos a respeito das eventuais consequências do seguimento ou do não seguimento de regras) e as consequências imediatas. Uma evidência disso é que o comportamento especificado pela regra continuou sendo mantido na Fase 3, mesmo quando essa correspondência deixou de existir, isto é, quando ele deixou de produzir as consequências imediatas (os pontos) prometidas na justificativa do Tipo 1. Assim, a história de controle pela justificativa do Tipo 2 ("Quando eu mostrar estes objetos para você, você deve fazer o seguinte:”) da Fase 2 pode ter contribuído para manter o seguimento de regra na Fase 3, na medida em que essa justificativa pode ter indicado que fazer o que a regra especificava era o correto, era o que deveria ser feito e, portanto, o que poderia produzir aprovação ou evitar eventuais desaprovações futuras.

Diferentemente, é possível que os Participantes $\mathrm{P} 14$ e P16 tenham seguido a regra na Fase 2 mais sob o controle das consequências imediatas programadas para o seguimento de regra do que das justificativas dos Tipos 1 e 2. Uma evidência disso é que, na Fase 3, quando o seguimento de regra deixou de produzir tais consequências imediatas, P14 e P16 deixaram de seguir a regra e passaram a apresentar um comportamen- 
to dependente de suas consequências imediatas, ou seja, a história de controle pelas consequências imediatas programadas para o seguimento de regra da Fase 2 pode ter contribuído para impedir a manutenção do seguimento de regra que não produzia tais consequências imediatas e para manter um comportamento dependente de suas consequências imediatas na Fase 3.

Os resultados da Fase 4 apresentam evidências empíricas adicionais de que o comportamento apresentado por P14 e P16 foi mantido dependentemente de consequências imediatas e independentemente de justificativas para o seguimento de regra e de que o comportamento apresentado por P11, P12, P13 e P15 foi mantido independentemente de tais consequências e dependentemente de justificativas do Tipo 2 para o seguimento de regra. Essa distinção funcional entre os comportamentos desses participantes é a distinção entre o comportamento controlado por regras (caso de P11, P12, P13 e P15) e o comportamento controlado por contingências de reforço (caso de P14 e P16; Albuquerque \& Paracampo, in press; Albuquerque et al., no prelo).

Quanto aos Participantes P17 e P18, poder-se-ia que dizer que P17 se comportou de forma similar à P11, P12, P13 e P15 até a Tentativa 59 da Fase 4 e que, de modo geral, o desempenho de P18 também é similar ao apresentado por P11, P12, P13 e P15, uma vez que todos esses cinco participantes apresentaram um comportamento independente de suas consequências imediatas nas Fases 3 e 4. Contudo, os dados mostrando que P17 e P18 seguiram regra nas Fases 2 e 3 e deixaram de seguir regra na Fase 4, sugerem que esses dois participantes responderam sob o controle de outras variáveis. P17 e P18 seguiram regra na Fase 3, possivelmente, não apenas devido à história de controle pela justificativa do Tipo 2, mas também devido à história de controle pela interação entre a justificativa do Tipo 1 e as consequências imediatas para o seguimento de regra. Desse modo, o tempo de exposição à discrepância entre essas variáveis (a justificativa do Tipo 1 e as consequências imediatas) nas Fases 3 e 4, pode ter contribuído para que o seguimento da regra discrepante tivesse deixado de ocorrer na Fase 4. Uma evidência disso é que os dois chegaram a ganhar pontos após terem deixado de seguir a regra. O que não está claro é por que P18, ao invés de continuar emitindo a sequência CEF (correta), apresentou a sequência CFE, formalmente similar à correta. Esse tipo de erro tem sido encontrado em outros estudos.

Em síntese, os resultados de seis de oito participantes do presente estudo, apoiam a proposição que sugere que a manutenção do seguimento de regras discrepantes depende, em parte, de duas características críticas da história do ouvinte. Uma dessas características é se o comportamento apresentado pelo ouvinte, antes de seu contato com a regra discrepante, é um comportamento mantido dependentemente de suas consequências imediatas. A outra característica é se esse comportamento é mantido independentemente de tais consequências. Com base em tais características históricas pode-se prever a probabilidade de o seguimento de regra discrepante vir a ser mantido ou não. Esse comportamento apresentado pelo ouvinte antes de seu contato com a regra discrepante, por sua vez, tende a ser mantido dependentemente de suas consequências imediatas, quando a sua manutenção é favorecida por uma história de controle por consequências imediatas para seguir (como a construída na Fase 2) e para não seguir regra (como a construída na Fase 3 ) e tende a ser mantido independentemente de suas consequências imediatas, quando a sua manutenção é favorecida por uma história de controle por justificativa do Tipo 2 (como a construída na Fase 2; Albuquerque \& Paracampo, 2017b; Albuquerque et al., no prelo). Pesquisas futuras poderiam fazer uma replicação sistemática do presente experimento com o objetivo de testar essa suposição sobre os efeitos dessa história de controle por justificativa do Tipo 2. Uma das vantagens da replicação sistemática é que os seus resultados são passíveis de serem comparados com os resultados dos estudos anteriores do mesmo programa de pesquisas relacionadas.

\section{Referências}

Albuquerque, L. C. (1991). Efeitos de regras no controle do comportamento humano [Resumo]. In Sociedade Brasileira de Psicologia (Ed.), Resumos de comunicações cientificas. XXI Reunião 
Anual de Psicologia (p. 162). Ribeirão Preto, SP: Sociedade Brasileira de Psicologia.

Albuquerque, L. C., de Souza, D. G., Matos, M. A., \& Paracampo, C. C. P. (2003). Análise dos efeitos de histórias experimentais sobre o seguimento subsequente de regras. Acta Comportamentalia, $11,87-126$.

Albuquerque, L. C., Mescouto, W. A., \& Paracampo, C. C. P. (2011). Controle por regras: Efeitos de perguntas, sugestões e ordens. Acta Comportamentalia, 19, 19-42.

Albuquerque, L. C., \& Paracampo, C. C. P. (2017a). Theory of control by justifications and immediate consequences. In J. C. Todorov (Ed.), Trends in Behavior Analysis: Vol. 2 (pp. 125-152). Brasília, DF: Technopolitik.

Albuquerque, L. C., \& Paracampo, C. C. P. (2017b). Control by stimuli constituent of rules and reinforcement contingencies. In J. C. Todorov (Ed.), Trends in Behavior Analysis: Vol. 2 (pp. 153191). Brasília, DF: Technopolitik.

Albuquerque, L. C., \& Paracampo, C. C. P. (in press). Selection of behavior by justifications constituent of rules. Trends in Psychology. Manuscript submitted for publication.

Albuquerque, L. C., Paracampo, C. C. P., \& Allan, S. (no prelo). Análise de variáveis que podem determinar as funções de regras e de contingências de reforço. Acta Comportamentalia. Manuscrito submetido para publicação.

Albuquerque, L. C., Paracampo, C. C. P., Matsuo, G. L., \& Mescouto, W. A. (2013). Variáveis combinadas, comportamento governado por regras e comportamento modelado por contingência. Acta Comportamentalia, 21, 285-304.

Albuquerque, L. C., Reis, A. A., \& Paracampo, C. C. P. (2006). Efeitos de uma história de reforço contínuo sobre o seguimento de regra. Acta Comportamentalia, 14, 47-75.

Albuquerque, L. C., Reis, A. A., \& Paracampo, C. C. P. (2008). Efeitos de histórias de reforço, curtas e prolongadas, sobre o seguimento de regras. Acta Comportamentalia, 16, 305-332.

Albuquerque, L. C., \& Silva, F. M. (2006). Efeitos da exposição a mudanças nas contingências sobre o seguir regras. Psicologia: Teoria e Pesquisa, 22, 101-112.

Albuquerque, L. C., Silva, L. S., \& Paracampo, C. C. P. (2014). Análise de variáveis que podem inter- ferir no comportamento de seguir regras discrepantes. Acta Comportamentalia, 22, 51-71.

Baum, W. M. (1999). Compreender o behaviorismo: Ciência, comportamento e cultura. Porto Alegre, RS: Artmed (Original publicado em 1994)

Catania, A. C. (1998). Learning. Hillsdale, NJ: Prentice Hall.

Cerutti, D. T. (1989). Discrimination theory of rulegovernedbehavior. Journal of the Experimental Analysis of Behavior, 51, 259-276.

Galizio, M. (1979). Contingency-shaped and rulegoverned behavior: Instructional control of human loss avoidance. Journal of the Experimental Analysis of Behavior, 31, 53-70.

Hayes, S. C., Brownstein, A. J., Zettle, R. D., Rosenfarb, I., \& Korn, Z. (1986). Rule governed behavior and sensitivity to changing consequences of responding. Journal of the Experimental Analysis of Behavior, 45, 237-256.

Malott, R. M. (1989). Achievement of evasive goals. In S. C. Hayes (Ed.), Rule governed behavior: Cognition, contingencies, and instructional control (pp. 153-190). New York: Plenum.

Martinez, H., \& Tomayo, R. (2005). Interactions of contingencies, instructional accuracy, and instructional history in conditional discrimination. The Psychological Record, 55, 633-646.

Matos, M. A. (2001). Comportamento governado por regras. Revista Brasileira de Terapia Comportamental e Cognitiva, 2, 51-66.

Matsuo, G. L., Albuquerque, L. C., \& Paracampo, C. C. P. (2014). Efeitos de justificativas relatadas em regras sobre o seguimento de regras. Acta Comportamentalia, 22, 273-293.

Newman, B., Buffington, D. M., \& Hemmes, N. S. (1995). The effects of schedules of reinforcement on instruction following. The Psychological Record, 45, 463-476.

Paracampo, C. C. P., \& Albuquerque, L. C. (2004). Análise do papel das consequências programadas no seguimento de regras. Interação em Psicologia, 8, 237-245.

Paracampo, C. C. P., Albuquerque, L. C., Mescouto, W. A., \& Farias, A. F. (2013). Efeitos de perguntas e de respostas às perguntas sobre o seguir regras. Psicologia: Teoria e Pesquisa, 29, 63-74.

Paracampo, C. C. P., Souza, L. M., \& Albuquerque, L. C. (2014). Variáveis que podem interferir no 
seguir regras de participantes flexíveis e inflexíveis. Psicologia: Reflexão e Crítica, 27, $124-$ 133.

Perez, W. F., Reis, M. J. D., \& de Souza, D. G. (2010). Efeitos de história experimental com diferentes instruções e do controle por contingências sobre o seguimento de instruções. Acta Comportamentalia, 18, 55-85.

Pinto, A. R., Paracampo, C. C. P., \& Albuquerque, L. C. (2006). Análise do controle por regras em participantes classificados de flexíveis e de inflexíveis. Acta Comportamentalia, 14, 171-194.

Rehfisch, J. M. (1958). A Scale for Personality Rigidity. Journal of Consulting Psychology, 1, 11-15.

Schlinger, H., \& Blakely, E. (1987). Function-altering effects of contingency-specifying stimuli. The Behavior Analyst, 10, 41-45. doi:10.1007/ BF03392405

Shimoff, E., Catania, A. C., \& Matthews, B. A. (1981). Uninstructed human responding: Sensitivity of low-rate performance to schedule contingencies. Journal of the Experimental Analysis of Behavior, 36, 207-220. doi:10.1901/jeab.1981.36-207

Skinner, B. F. (1969). Contingencies of reinforcement: A theoretical analysis. New York: Appleton-Century-Crofts.
Skinner, B. F. (1974). About behaviorism. New York: Alfred A. Knopf.

Torgrud, L. J., \& Holborn, S. W. (1990). The effects of verbal performance descriptions on nonverbal operant responding. Journal of the Experimental Analysis of Behavior, 54, 273-291. doi:10.1901/ jeab.1990.54-273

Wulfert, E., Greenway, D. E., Farkas, P., Hayes, S. C., \& Dougher, M. J. (1994). Correlation between self-reported rigidity and rule-governed insensivity to operant contingencies. Journal of Applied Behavior Analysis, 27, 659-671. doi:10.1901/jaba.1994.27-659

Zettle, R. D., \& Hayes, S. C. (1982). Rule-governed behavior: A potential theoretical framework for cognitive-behavior therapy. In P. C. Kendall (Ed.), Advances in cognitive-behavioral research and therapy (pp. 73-118). New York: Academic Press.
Recebido: 12/02/2016

$1^{a}$ revisão: $11 / / 11 / 2016$

$2^{a}$ revisão: $25 / 11 / 2016$

Aceite final: 25/11/2016 